Sir,

\section{Corneal involvement in xanthoma disseminatum}

Cutaneous xanthomata are well-circumscribed yellow or brown papules, nodules or plaques that may be associated with hyperlipidaemia or neoplastic proliferation of histiocytes. Xanthoma disseminatum is a rare histiocytosis syndrome with widespread involvement of the skin, mucous membranes and central nervous system. We describe a case of xanthoma disseminatum with bilateral lid and corneal involvement.

\section{Case report}

A 13-year-old boy of Pakistani origin with known xanthoma disseminatum was referred for ophthalmological assessment. There was no family history of the condition and no history of consanguinity in his parents. At 11 years of age he developed extensive cutaneous xanthomata that rapidly increased in number becoming confluent at the skin flexures (Fig. 1). Serum lipid profiles were normal. Cerebral involvement had caused temporal lobe epilepsy, diabetes insipidus and

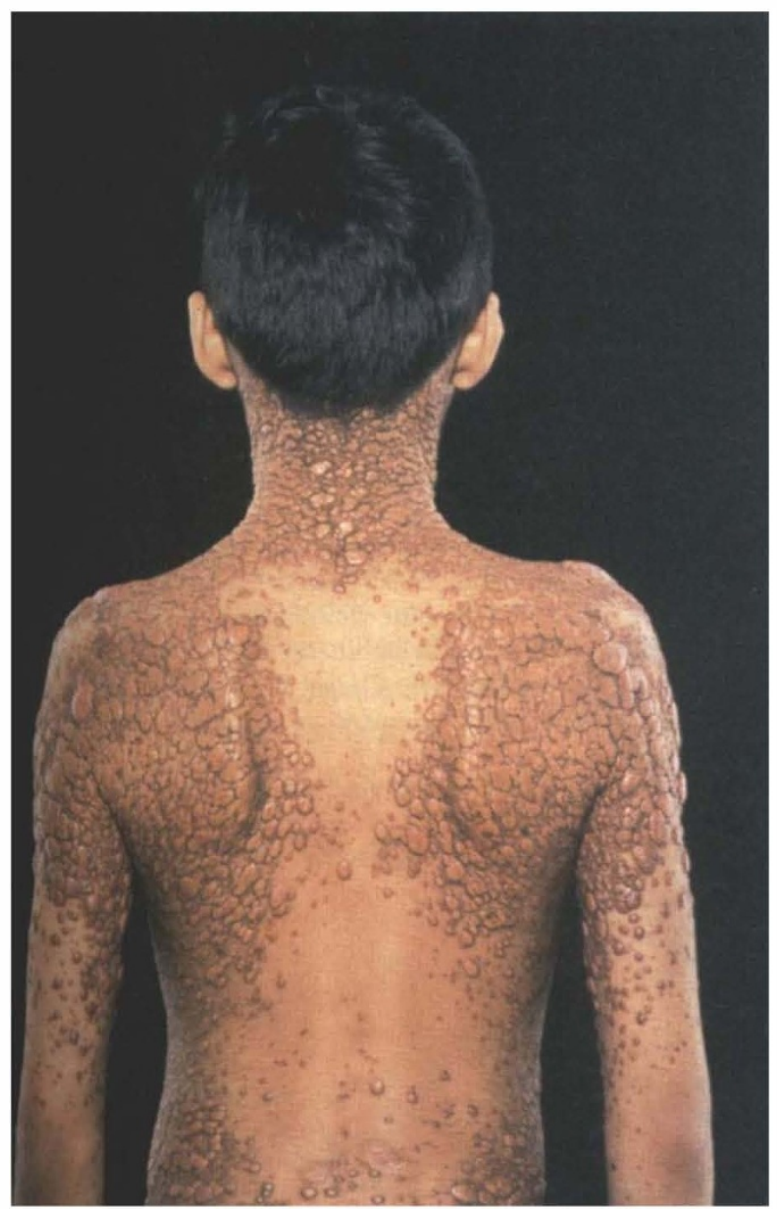

Fig. 1. The cutaneous lesions of xanthoma disseminatum.

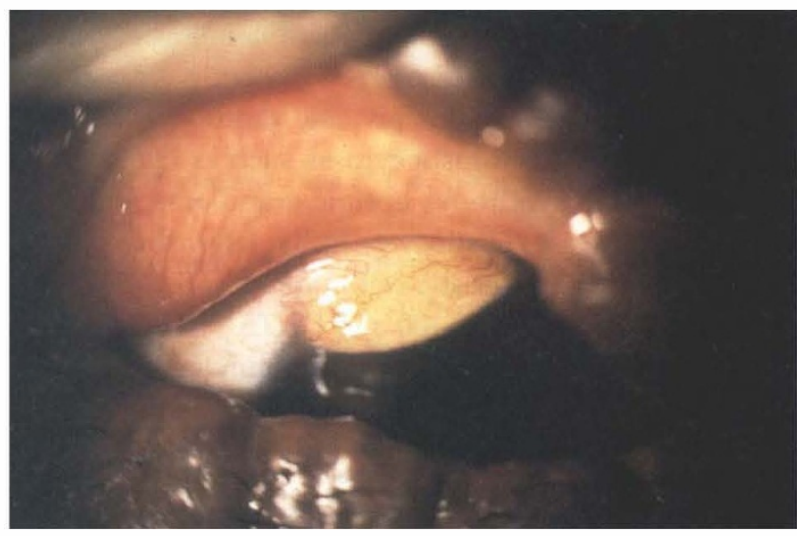

Fig. 2. Superior limbal xanthoma of the right eye.

generalised hypopituitarism with retarded growth, hypothyroidism, delayed puberty and small testes. Magnetic resonance imaging of the head confirmed lesions in the region of the pituitary gland. Multiple endocrine replacement therapy with growth hormone, thyroxine, androgen and vasopressin had been instituted. Numerous xanthomata were present in the larynx and pharynx producing stenosis of the upper airway with stridor and dyspnoea on exertion. Laser division of the supraglottis had been performed with some improvement of his symptoms.

The lids were extensively involved with small xanthomata. Solitary smooth, yellow slightly raised nodules were present at the superior limbus of both eyes extending onto the cornea for approximately $1 \mathrm{~mm}$ (Fig. 2). The eyes were comfortable with $6 / 6$ vision right and left. There was no evidence of iris, ciliary body or chorioretinal xanthomata.

Histological examination of skin and laryngeal biopsies showed large numbers of foamy histiocytes with some giant cells of the Touton type. There was no binding with S-100 and OKT6 monoclonal antibodies, confirming that these cells were not Langerhans cells.

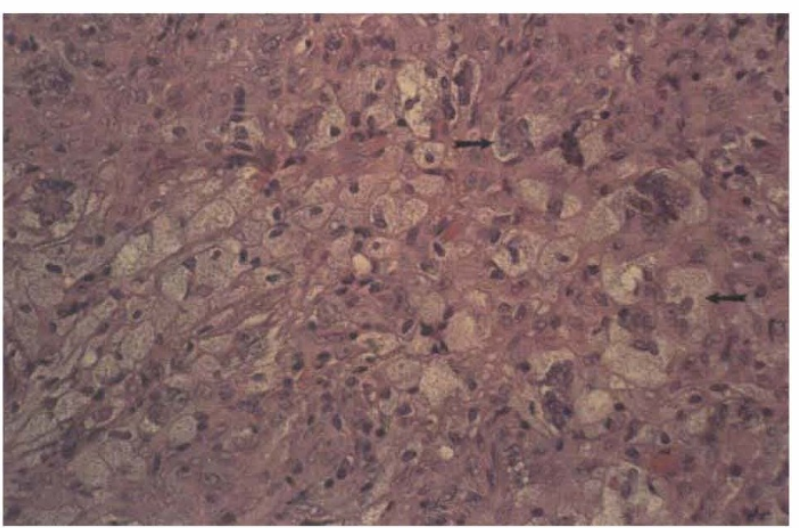

Fig. 3. Photomicrograph of a histological section through a laryngeal xanthoma $(\times 125)$ showing giant cells of the Touton type (arrowed). 
The extracellular matrix showed extensive fibrosis (Fig. 3). The ocular lesions were not biopsied.

\section{Discussion}

Xanthoma disseminatum belongs to a varied group of syndromes that have in common a proliferation of mononuclear phagocytes (histiocytes). Other members of this group of diseases include juvenile xanthogranuloma and the Langerhans cell histiocytoses such as HandSchuller-Christian syndrome, eosinic granuloma and Letterer-Siwe disease. Histiocytes in the latter three conditions bind characteristic cell markers for Langerhans cells such as S-100 and OKT6. ${ }^{1}$

The histological features of the xanthomata are identical in xanthogranuloma disseminatum and juvenile xanthogranuloma. Differentiation of these nonLangerhans cell histiocytoses therefore depends upon the clinical pattern and severity of organ involvement. ${ }^{2,3}$

Xanthoma disseminatum is an unusual sporadic syndrome characterised by widespread involvement of the skin, mucosal surfaces and central nervous system.' The onset is usually in later childhood or young adulthood. As in our patient, involvement of the pituitary axis may lead to multiple endocrine abnormalities, and diabetes insipidus occurs in $40 \%$ of patients. Meningeal lesions can produce epilepsy.

Lesions in the respiratory mucosa can give rise to stridor and dyspnoea. Large areas of the body may be covered by red-yellow cutaneous xanthomata that have a predilection for the skin flexures and eyelids. As the skin lesions mature they often become brown in colour. Xanthoma disseminatum usually has a benign course and resolution after a few years is common. ${ }^{1}$ Despite this, significant morbidity and even mortality can occur in active disease from neurological and upper airway complications. Corneal or conjunctival xanthomata occur in $20 \%$ of patients with xanthoma disseminatum. Corneal xanthomata have also been reported in juvenile xanthogranuloma but are unusual. ${ }^{4,7}$ Iris and ciliary body xanthomata are the most frequent ocular manifestation of juvenile xanthoma (occurring in 10\% of cases) and have not to our knowledge been reported in xanthoma disseminatum. They appear as localised or diffuse yellowish iris lesions that may only be discovered because of secondary effects such as spontaneous hyphaema or unilateral glaucoma. ${ }^{5}$

An effective systemic therapy for xanthoma disseminatum has not been found. Vincristine, steroids, cyclophosphamide, chlorambucil, methotrexate, 5 -fluorouracil and azathioprine have all been tried with very limited response. ${ }^{1}$ Localised cryotherapy using a freeze-thaw-freeze technique has been advocated for skin lesions with some success. ${ }^{6}$ The poor response to irradiation and chemotherapy is in contradistinction to the improvement seen with these modalities in the Langerhans cell histiocytoses.

Lamellar sclerokeratectomy has been successful in removing limbal xanthomata in juvenile xanthogranuloma and those occurring as isolated lesions. ${ }^{7-10}$ The xanthomata may occupy most of the corneal stroma and the resulting corneal defect may require lamellar keratoplasty. ${ }^{4}$ The xanthomata often extend across the limbus deep into the sclera making complete excision difficult, and recurrences may occur. ${ }^{10}$ There is less in the literature regarding the treatment of limbal xanthomata occurring as part of xanthoma disseminatum. One of the patients described by Liebman et al. ${ }^{4}$ in 1966 represented a case of corneal xanthomata in xanthoma disseminatum. The corneal lesions were treated with radiotherapy via a strontium-90 applicator and surgical excision. Despite this, the lesions progressed, requiring further excision and irradiation. The outcome was poor with total corneal opacification and thinning leading to profound visual loss in one eye and only a small central window of relatively clear cornea remaining in the other eye. Interestingly the skin, mouth and pharyngeal lesions almost completely disappeared in this patient in later childhood.

Excision and irradiation carry the risks of dry eye, vascularisation, thinning and progressive corneal opacification. In our patient the limbal xanthomata have not progressed and the visual acuity remains good. Fortunately the prognosis in xanthoma disseminatum is good and spontaneous resolution is usual. We recommend that unless significantly interfering with vision, corneoscleral lesions in xanthoma disseminatum are probably best left alone.

\section{References}

1. Giller RH, Folberg R, Keech RG, Piette WW, Sato Y. Xanthoma disseminatum: an unusual histiocytosis syndrome. Am J Pediatr Haematol Oncol 1988;10:252-7.

2. Favara BE. The pathology of 'histiocytosis'. Am J Pediatr Haematol Oncol 1981;3:45-56.

3. Writing Group of the Histiocytosis Society. Histiocytosis syndrome in children. Lancet 1987;1:208-9.

4. Liebman SD, Crocker AC, Greiser CF. Corneal xanthoma in childhood. Arch Ophthalmol 1966;76:221-9.

5. DeBarge LR, Chan CC, Greenberg SC, McLean IW, Yannuzzi LA, Nussenblatt RB. Chorioretinal, iris and ciliary body infiltration by juvenile xanthogranuloma masquerading as uveitis. Surv Ophthalmol 1994;39:65-71.

6. Kavalec CC, Harvey JT. Xanthoma disseminatum: photo essay. Arch Ophthalmol 1993;111:1428-9.

7. Yanoff M, Perry HD. Juvenile xanthogranuloma of the corneoscleral limbus. Arch Ophthalmol 1995;113:915-7.

8. Harvey P, Lee JA, Talbot JF, Goepel JR. Isolated xanthogranuloma of the limbus in an adult. Br J Ophthalmol 1994;78:657-9.

9. Grayson M, Pieroni D. Solitary xanthoma of the limbus. Br J Ophthalmol 1970;54:652-4.

10. Collum LM, Mullaney J. Adult limbal xanthogranuloma. Br J Ophthalmol 1984;68:360-3.

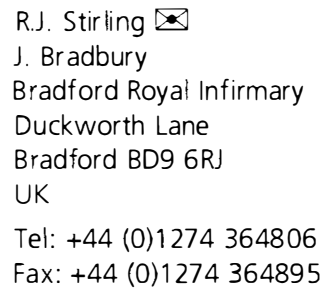

